\title{
Accuracy Analyses of Synthesized- Reference-Wave Holography for Determining Antenna Radiation Characteristics
}

\author{
Vladimir Schejbal ${ }^{1}$, Jan Pidanic ${ }^{1}$, Vlastimil Kovarik $^{2}$, and Dusan Cermak ${ }^{1}$ \\ ${ }^{1}$ University of Pardubice, Jan Perner Transport Faculty \\ Studentska 95, 53210 Pardubice, Czech Republic \\ E-mail: vladimir.schejbal@upce.cz; jan.pidanic@upce.cz; dusan.cermak@upce.cz \\ ${ }^{2}$ Retia, a.s. \\ Prazska 341, Zelene Predmesti, 53002 Pardubice, Czech Republic \\ E-mail: vkovarik@retia.cz
}

\begin{abstract}
Synthesized-reference-wave holographic techniques offer relatively simple and cost-effective measurements of antenna radiation characteristics, and reconstruction of complex aperture fields using near-field intensity-pattern measurements. These methods allow utilization of the advantages of the methods for probe compensation for near-field amplitude and phase measurements for planar and cylindrical scanning, including accuracy analyses. Accuracy analyses using mathematical models considering random processes with correlation intervals are presented. Numerical simulations, taking into account random as well as deterministic errors and the processing of measurement statistics, are also presented. It is demonstrated that the given analyses correspond to our measurements and/or numerical simulations.
\end{abstract}

Keywords: Antenna measurements; antenna radiation patterns; accuracy analyses; near-field sampling; holography; nearfield measurements; antenna probe compensation

\section{Introduction}

$\mathbf{N}$ ear-field (NF) measurements provide a fast and accurate method for determining an antenna's gain, pattern, polarization, beam pointing, etc. In contrast to conventional far-field methods, near-field antenna measurement methods make use of a measuring probe in the radiating near-field region of the antenna under test (AUT). The far-field radiation pattern of the AUT must then be indirectly computed from the measurements made in a near-field region. Several methods for near-field antenna measurements have been described, such as [1-17].

Synthesized-reference-wave holography was proposed in the $\mathrm{PhD}$ thesis [9], where several aspects were thoroughly analyzed. (These methods allow employing the advantages of methods for probe compensation for amplitude and phase near-field measurements for planar and cylindrical scanning, including accuracy analyses [1-7]). Most of the results have been published [10-16], such as a description of holographic near-field measurements using probe compensation for planar and cylindrical scanning; a comparison of holographic near-field and far-field measurements with and without probe compensation; accuracy analyses using mathematical models considering random processes with correlation intervals; numerical simulations considering random errors as well as deterministic errors; and the processing of measurement statistics. Paper [17] summarized the cited results (as some of the papers and the $\mathrm{PhD}$ thesis were only in (zech), and gave some new results (especially numerical simulations). This paper analyzes the accuracy, using mathematical models considering random processes with correlation intervals, the processing of measurement statistics, and numerical simulations considering random as well as deterministic errors. The analyses given correspond to our measurements and/or numerical simulations.

\section{Accuracy Analyses}

Near-field measurements using synthesized-reference-wave holography with probe compensation for planar and cylindrical scanning allow employing the advantages of methods for probe compensation for the amplitude and phase of near-field measurements for planar and cylindrical scanning, including accuracy analyses [1-7], which should be slightly modified. However, accuracy analyses using mathematical models considering random processes with correlation intervals, measurements using statistical processing, and numerical simulations considering random as well as deterministic errors have been done, and they are described below. 
The theory of near-field antenna measurements applies rigorously to linear antennas. In the theory of probe-corrected near-field measurements, multiple reflections between the probe and test antennas are considered negligible, but experimental errors also limit the accuracy of near-field techniques. The experimental measurements introduce probe-positioning errors, instrumentation errors, and - for the planar and cylindrical (or truncated spherical) scanning geometries - finite scanning errors. Errors are also created by room reflections, uncertainties in the far field of the probe, and uncertainties in the measurement of the insertion loss between the test antenna and the probe when absolute gain is required. The NIST 18-term error model can be applied [4, 7]. If the sample spacing and computer accuracy are adequate, aliasing and computational errors should be negligible compared to the experimental errors. The importance of the various near-field measurement errors depends on the antenna under test, the frequency of operation, the measurement facility, and the probe. However, results show that the finite scanning area, the $z$ position of the probe for planar scanning, receiver nonlinearities in measuring the near-field amplitude, and, sometimes, multiple reflections are usually the most important sources of error.

In general, planar scanning is limited to determining the fields within the forward solid angular region subtended by the edges of the test antenna and the finite scanning area. Cylindrical scanning omits only the biconical angular region formed by the outer edges of the test antenna and the cylindrical scanning area of finite height. It is possible to use a priori information on the geometry of the antenna under test (AUT) and to evaluate the farfield pattern from the estimated near-field data. The method of [3] takes advantage of the possibility - present in most of the scanning setups - of moving the probe not only on the scanning surface, but also along the axis perpendicular to the scanning surface. These effects are the same, even for synthesized-reference-wave holography [17].

The $z$-position inaccuracies, i.e., the deviation, $\delta$, from planarity of the probe transport over the scanning area, can produce relatively large errors in the sidelobe levels of the far field. Variations in the $z$ position of the probe produce corresponding variations in the near-field phase. Thus, large errors in the sidelobes occur in the far-field directions corresponding to the predominant spatial frequencies of the variation in $z$ position across the scanning area. In the main-beam direction, this is much less critical: the reduction in gain is given by $\delta^{2} / 2$, from the familiar Ruze relation [1]. The errors in the sidelobes caused by inaccurate $z$ positioning can be reduced by measuring the deviation of the probe from the scanning plane and correcting the near-field phase. It should also be mentioned that receiver phase errors generally have a much smaller effect on the far field than do phase errors caused by inaccurate $z$ positioning. This is because typical receiver phase errors are negligible at the maximum near-field amplitude, and increase monotonically with decreasing amplitude. However, receiver nonlinearities in the measurement of near-field amplitude can cause significant errors in the main beam and sidelobes of the far fields. The contribution to the output of the probe from the multiple reflections can be estimated by changing the separation distance between the probe and test antenna, and recording the amplitude variations that occur in the received signal with a period of about $\lambda / 2$. If multiple reflections prove significant, they may be reduced by the sensible use of absorbing material, by decreasing the size of the probe, by increasing the probe separation distance, by averaging the far fields computed from the near-field data taken on scanning planes that are separated by a small fraction of a wavelength (about $\lambda / 4$ or less), or by using specially designed probes that filter the main beam and emphasize the sidelobes.

However, the upper-bound error formulas should be applied with care. They are dependent upon underlying (usually, explicitly stated) assumptions that are satisfied by most antennas and nearfield measurement conditions, but which may not be fulfilled in certain circumstances. For example, it is well known that phase errors introduced into the main near-field beam of directive antennas cause a reduction in the computed on-axis gain. However, this gain reduction strictly applies to near-field beams of uniform phase. When the underlying assumptions can be relaxed, lower values for upper bounds can usually be obtained. For example, an estimate of the specific $z$-position errors for a particular measurement facility allows one to more accurately estimate their effect upon far-field sidelobes.

Accuracy analyses using mathematical models considering random processes with correlation intervals have been performed $[9,11]$ using the results of $[8,19]$. Therefore, only a brief summary is given. The measurement scanning [17] is done on a finite surface, $S$, and the most important part of the power radiates through that surface. The surface $S$ can be transformed onto a square region, $[-1,1] \times[-1,1]$, and normalized coordinates $x_{1}, x_{2}$ could be used for both planar and cylindrical scanning. If the theoretically measured values are $v\left(x_{1}, x_{2}\right)$ and the values affected by errors are $v_{F}\left(x_{1}, x_{2}\right)$, then the following relationship can be considered:

$$
v_{F}\left(x_{1}, x_{2}\right)=v\left(x_{1}, x_{2}\right)\left[1+B\left(x_{1}, x_{2}\right)\right] e^{j q\left(x_{1}, x_{2}\right)},
$$

where the values of $B\left(x_{1}, x_{2}\right)$ are the amplitude errors, and the values of $q\left(x_{1}, x_{2}\right)$ are the phase errors. As requirements on antennaparameter measurements are usually quite strict, only the case of small errors (i.e., $\left.\left|B\left(x_{1}, x_{2}\right)\right|,\left|q\left(x_{1}, x_{2}\right)\right| \ll 1\right)$ can be considered. The random errors, $\left|B\left(x_{1}, x_{2}\right)\right|$ and $q\left(x_{1}, x_{2}\right)$, are assumed to have normal distributions, zero means, constant variances given by $\sigma_{B}^{2}$ and $\sigma_{q}^{2}$ (i.e., $\sigma_{B}^{2}, \sigma_{q}^{2}$ do not depend on $x_{1}, x_{2}$ ), and correlation coefficients $r_{B}$ and $r_{q}$. The following correlation coefficients are considered:

$$
r_{B}\left(X_{1}, X_{2}\right)=e^{-\left(\frac{x_{1}^{2}}{c_{1}^{2}}+\frac{X_{2}^{2}}{c_{2}^{2}}\right)},
$$

where $c_{1}$ and $c_{2}$ are correlation intervals, $X_{1}=x_{1}-\eta_{1}$ and $X_{2}=x_{2}-\eta_{2}$ are differences, and $x_{1}$ and $x_{2}$ are normalized coordinates. Similarly, the correlation coefficient $r_{q}\left(X_{1}, X_{2}\right)$ can be used for phase errors.

The radiation patterns are completely described by angular wave spectra $A\left(K_{1}, K_{2}\right)$, which are given by the Fourier transform of the measured field, $\mathbf{F}\left[v\left(x_{1}, x_{2}\right)\right]$, and similarly for $v_{F}\left(x_{1}, x_{2}\right)$. It should be noted that the surface $S$ is transformed, as well as $K_{1}$, $K_{2}$, and $A\left(K_{1}, K_{2}\right)$. It is well known (the scaling theorem) that

$$
\mathbf{F}\left[v\left(l_{1} v_{1}, l_{2} v_{2}\right)\right]=A\left(K_{1} / l_{1}, K_{2} / l_{2}\right) /\left(l_{1} l_{2}\right)
$$


Considering the assumptions of synthesized-reference-wave holography [17], the following equation can be derived for a suitable interval $\left|k_{x}\right|<k_{1}$ :

$$
\begin{aligned}
\mathbf{F}\left[v\left(x_{1}, x_{2}\right) e^{-j a x_{1}}\right] \\
=\mathbf{F}\left[\left|E\left(x_{1}, x_{2}\right)\right|^{2} e^{-j a x_{1}}+||^{2} e^{-j a x_{1}}\right. \\
\left.\quad+C^{*} E\left(x_{1}, x_{2}\right)+C E^{*}\left(x_{1}, x_{2}\right) e^{-2 j a x_{1}}\right] \\
=\mathbf{F}\left[C^{*} E\left(x_{1}, x_{2}\right)\right],
\end{aligned}
$$

where $C \exp \left(-j a x_{1}\right)$ is the reference signal, and $E(x)$ is the near field.

The following expression is valid (the bars denote the mean values)

$$
\overline{A_{F}\left(K_{1}, K_{2}\right)} \dot{\doteq}\left(1-\frac{\sigma_{q}^{2}}{2}\right) A\left(K_{1}, K_{2}\right)
$$

where the higher-power functions of $\sigma_{q}$ have been neglected. This means that the mean value of the angular spectra of $v_{F}\left(x_{1}, x_{2}\right)$ are equal to the angular spectra of the original field $v\left(x_{1}, x_{2}\right)$, except for a multiplicative constant.

The variances of the angular spectra are $[8,19]$

$$
\overline{\left|\Delta A\left(K_{1}, K_{2}\right)\right|^{2}}=\alpha_{B} \sigma_{B}^{2}+\alpha_{q} \sigma_{q}^{2} \text {, }
$$

where the coefficients $\alpha_{B}$ and $\alpha_{q}$ determine the effects of the individual $B\left(x_{1}, x_{2}\right)$ and $q\left(x_{1}, x_{2}\right)$ errors.

If the individual errors are considered to be independent errors and $c_{1}, c_{2} \ll 1$, then $[8,19]$

$$
\begin{aligned}
& \alpha_{B}\left(K_{1}, K_{2}\right) \sigma_{B}^{2} \\
& \doteq \frac{1}{(2 \pi)^{2}} \int_{-1}^{1} \int_{-1}^{1} \int_{-1-1}^{1} \int_{-1}^{1} v\left(x_{1}, x_{2}\right) v^{*}\left(\eta_{1}, \eta_{2}\right) \sigma_{B}^{2} r_{B}\left(X_{1}, X_{2}\right) \\
& e^{-j\left(K_{1} X_{1}+K_{2}, X_{2}\right)} d x_{1} d x_{2} d \eta_{1} d \eta_{2} \\
& \doteq \pi c_{1} c_{2} e^{-\left[\left(K_{1}^{2} c_{1}^{2}+K_{2}^{2} c_{2}^{2}\right) / 4\right]} \frac{\sigma_{B}^{2}}{(2 \pi)^{2}} \int_{-1-1}^{1} \int\left|v\left(x_{1}, x_{2}\right)\right|^{2} d x_{1} d x_{2} \\
& =\frac{c_{1} c_{2} \sigma_{B}^{2}}{4 \pi} e^{-\left[\left(K_{1}^{2} c_{1}^{2}+K_{2}^{2} c_{2}^{2}\right) / 4\right]} \int_{-\infty}^{\infty} \int_{-\infty}^{\infty}\left|A\left(K_{1}, K_{2}\right)\right|^{2} d K_{1} d K_{2} .
\end{aligned}
$$

Similarly,

$$
\begin{aligned}
& \alpha_{q}\left(K_{1}, K_{2}\right) \sigma_{q}^{2} \\
& \quad \doteq \frac{c_{1} c_{2} \sigma_{q}^{2}}{4 \pi} e^{-\left[\left(K_{1}^{2} c_{1}^{2}+K_{2}^{2} c_{2}^{2}\right) / 4\right]} \int_{-\infty}^{\infty} \int_{-\infty}^{\infty}\left|A\left(K_{1}, K_{2}\right)\right|^{2} d K_{1} d K_{2} .
\end{aligned}
$$

The variances of the angular spectra are given by Equation (6). The individual terms, $\alpha_{B} \sigma_{B}^{2}$ and $\alpha_{q} \sigma_{q}^{2}$, are given by Equations (7) and (8). This allows the determination of the variances of the angular spectra for individual errors.

The random-error assumptions used generally cannot be fulfilled, but they are fulfilled for various important cases. This could simplify the analyses of random-error effects (quite general assumptions would not allow any analysis). It was shown [19] that nearly the same results can be obtained if $r_{B}$ (or, possibly, $r_{q}$ ) is given not by Equation (2), but it is only required that the correlation coefficients decrease monotonically with increasing $\left|X_{1}\right|$ and/or $\left|X_{2}\right|$. This means that even if the above assumptions are not exactly fulfilled, Equations (5) to (8) are approximately valid.

Other parameter changes were analyzed [19], such as the extremes of the angular-spectrum mean values, and the direction changes for angular-spectrum maximum and beam-width changes. Linear and surface apertures were also compared. It is clear that the requirements on the measurement accuracy for a linear antenna aperture are higher than the requirements for two-dimensional apertures. This follows from the derivation of Equations (5) and (6) for the one-dimensional case. This could be explained by considering the fact that statistical analyses are performed for two coordinates for the two-dimensional case, whereas for linear antennas they are performed only for one coordinate.

All given equations correspond to the case where radiation patterns are determined using one realization of near-field measurements. If the mean values are calculated using $n$ realizations, then the angular-spectrum variances are reduced $n$ times, i.e., the standard deviation by the $\sqrt{n}$ times.

The computational accuracies are very high and they should not be considered if the accuracies of the measurement are considered. The above analyses could be applied for generator-frequency instabilities, which change the phase differences between the direct and reference signals, as well as for generator-amplitude instabilities and scalar receiver (or possibly power-meter) errors. Similarly, they can be used for probe-position inaccuracies.

Periodic errors can be created during scanning. The phase shifters do not change phases in an arbitrary range, but only for the interval of $[0,2 \pi]$. That means only the values $\Phi-2 m \pi$ are set (where $m$ is an integer), instead of $\Phi$. Systematic phase-shifter errors create periodic errors, the effects of which have been analyzed by several authors, such as in [20]. If the errors are $\Delta_{s}=\beta \sin \Phi$, where $\Phi=-a x$ is the required phase value, then the following equation is valid for the interval $\left|k_{x}\right|<k_{1}$ :

$$
\mathbf{F}\left[v_{s}\left(x_{1}\right) e^{-j a x_{1}}\right] \doteq \mathbf{F}\left[C^{*} E\left(x_{1}\right)\right]+\frac{\beta}{2} \mathbf{F}\left[C E^{*}\left(x_{1}\right) e^{-3 j a x_{1}}\right],
$$

since the other terms are equal to zero for the interval $\left|k_{x}\right|<k_{1}$. The span of the spatial period $N \Delta k_{1}$ determines if the last term will appear in the interval $\left|k_{x}\right|<k_{1}$ (see [17] for details).

The selection of $\Delta x$ or $\Delta z$ scanning for the holographic method is a difficult problem, as this determines the angular space [17], and therefore the measurement accuracy. 


\section{Measurements}

We have done statistical processing of measurements $[9,15]$. Near-field measurements performed by line scanning take less time than those performed by planar or cylindrical scanning. Therefore, line scanning can be performed many times, and statistical methods can be used to support both the analyses of the accuracy of the holographic method for measuring near-field microwave antenna parameters, and the results of numerical simulations.

We chose a slot antenna (a waveguide with five slots in the broad wall, terminated by a matched load) for the measurement. Measurements were carried out with the reference polarization at a wavelength of $\lambda=32 \mathrm{~mm}$. The measuring arrangement was described in detail in $[9,13]$. Every measurement was performed by line scanning with 64 samples. After taking a fast Fourier transform (FFT), 64 values of the angular spectrum could be found. A maximum of one-third of this number, i.e., 21 values, could be used. These corresponded to the values of the angular spectrum (Fourier transform), $\mathbf{F}\left[C^{*} E(x)\right]$.

Ten measurements were carried out with a dipole probe and a spacing of $\Delta x=5 \mathrm{~mm}$, with $x$ values ranging from $-160 \mathrm{~mm}$ to $155 \mathrm{~mm}$, i.e., $N=64$ samples. The measured values (Meas. 1, Meas. 5, and Meas. 9) are shown in Figure 1. The other measurements were omitted for the sake of clarity. The angular-spectrum values of these measurements are shown in Figure 2 (to be more accurate, these are the amplitudes in $\mathrm{dB}$, normalized to the first angular-spectrum value). For clarity, only the angular-spectrum values corresponding to the measurements in Figure 1 are shown.

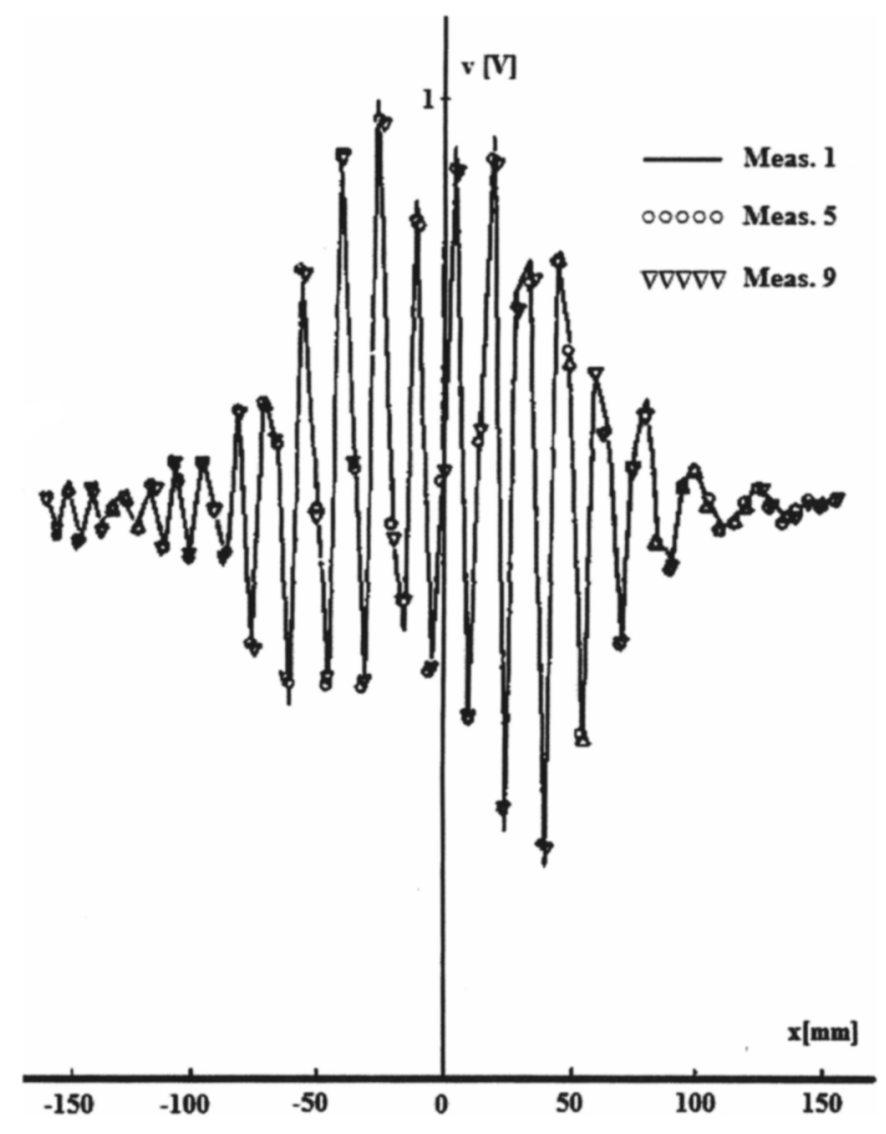

Figure 1. A measurement with a dipole probe, $\Delta x=5 \mathrm{~mm}$, $a \Delta x=2 \pi / 3$, along a straight line.

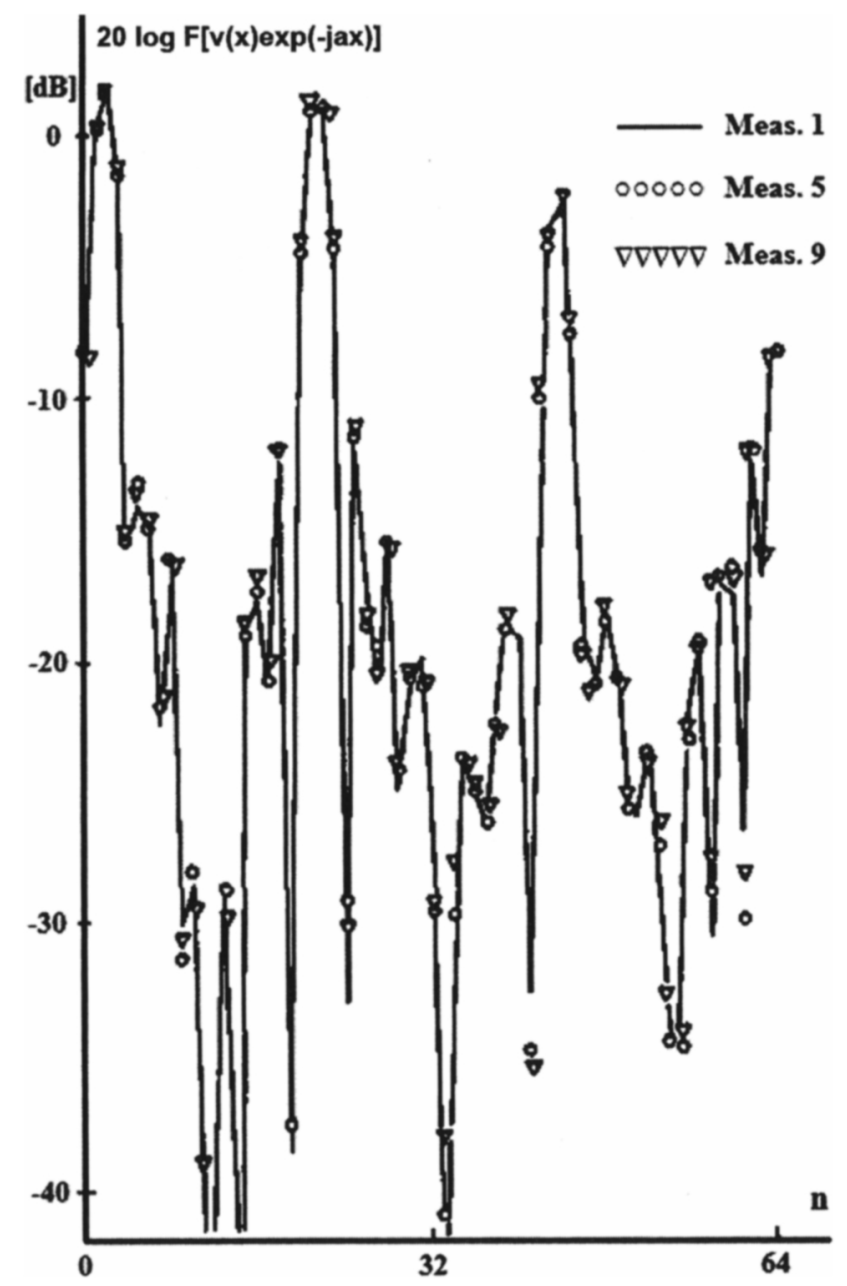

Figure 2. The values of the angular spectrum, $20 \log \mathbf{F}\left[v(x) e^{-j a x}\right]$, measured with a dipole probe using $\Delta x=5 \mathrm{~mm}$.

When testing the statistical hypothesis that the variance, $\sigma^{2}$, of a random variable, $x$, is $\sigma^{2}=\sigma_{0}^{2}$, the critical value of the $\mathrm{F}$ distribution and a suitable level of significance are considered. The sample mean,

$$
\bar{x}=\frac{1}{n} \sum_{i=1}^{n} x_{i},
$$

and sample variance,

$$
S^{2}=\frac{1}{n-1} \sum_{i=1}^{n}\left(x_{i}-\bar{x}\right)^{2},
$$

for random samples $x_{1}, x_{2}, \ldots, x_{n}$ can be calculated.

The sample mean values, Equation (10), of the angular spectrum using 10 measurements are plotted in Figure 3. The mean values were normalized to the maximum mean of the angular spectrum. The mean values of the angular spectrum, measured with a dipole probe and $\Delta x=5 \mathrm{~mm}$ spacing, are shown as a solid line. Each of the values was plotted as a function of the angle, $\vartheta$. For comparison, the radiation pattern measured in the far field is shown by a dashed line. The radiation pattern differs from the angular spectrum (see [1]), yet it is evident that in the vicinity of the main lobe, the far-field measurements and the near-field measurements 
obtained by the dipole probe using a step size of $\Delta x=5 \mathrm{~mm}$ are in good agreement.

To show the influence of an incorrect choice of the spacing $\Delta x$, ten measurements were performed with the dipole probe, a spacing $\Delta x=7.5 \mathrm{~mm}$, and a number of samples $N=64$, i.e., $\Delta x$ ranged from $-240 \mathrm{~mm}$ to $232.5 \mathrm{~mm}$. In this case, the individual components of the angular spectrum affected each other (aliasing). The sample mean values of these measured values are shown as circles in Figure 3. Ten measurements were carried out to show the influence of the probe. An open-ended $5 \times 22.86 \mathrm{~mm}$ waveguide was used as a probe, with a step size of $\Delta x=5 \mathrm{~mm}$, and $N=64$ samples, i.e., $\Delta x$ ranged from $-160 \mathrm{~mm}$ to $155 \mathrm{~mm}$. The angular spectrum of the probe will change the resulting spectrum, as followed from [1]. Therefore, a probe correction is necessary for computing radiation patterns. The sample mean values of these measurements are plotted as crosses in Figure 3.

The sample variances of the angular spectrum were calculated from Equation (11) using 10 measurements every time. The

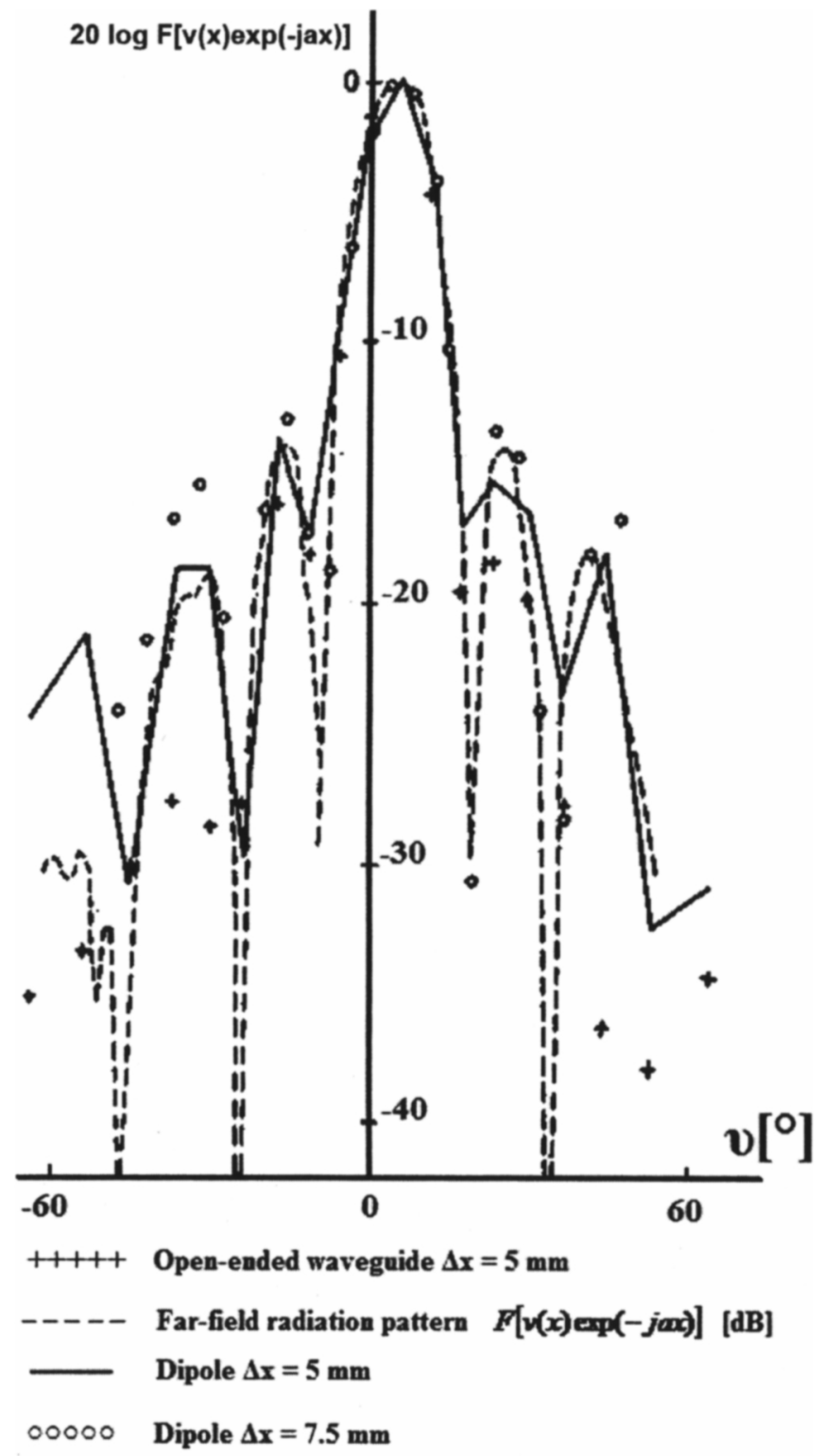

Figure 3. The sample means of the angular spectrum, $20 \log \mathbf{F}\left[v(x) e^{-j a x}\right]$.

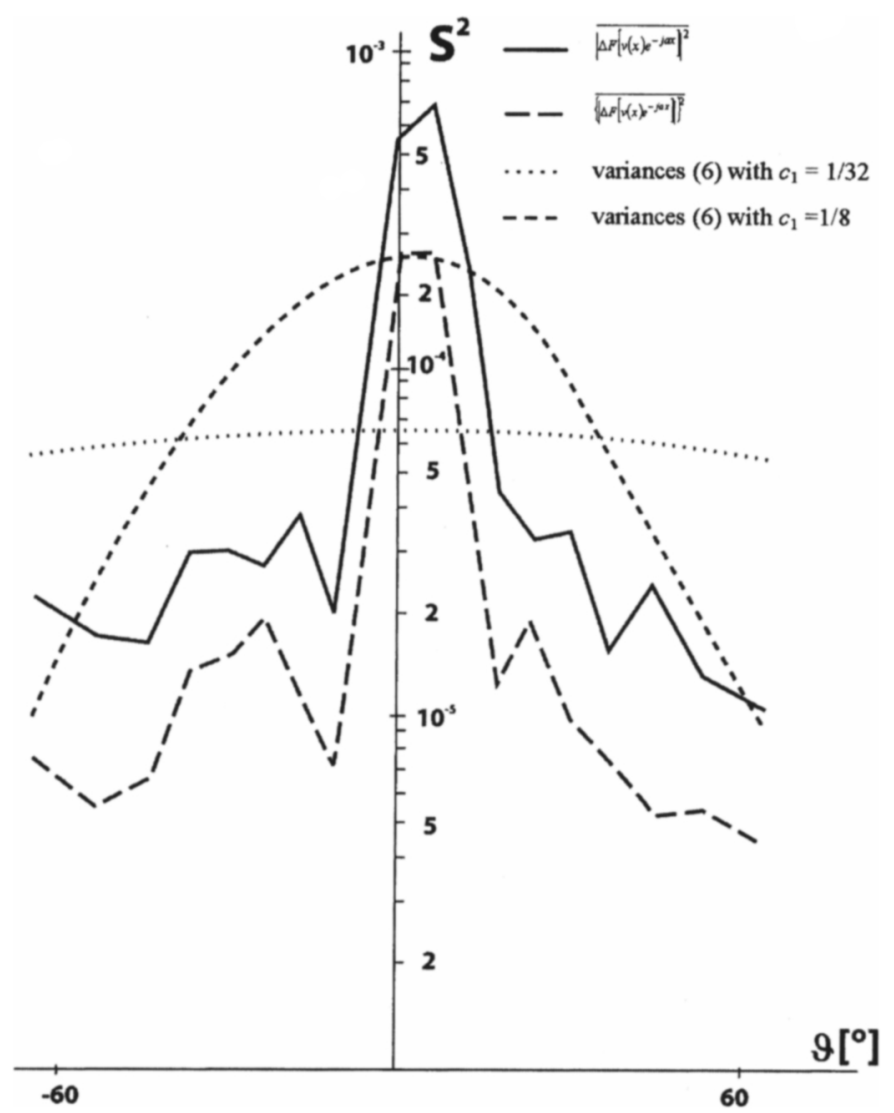

Figure 4. The variances of the measurements with a dipole, $\Delta x=5 \mathrm{~mm}$.

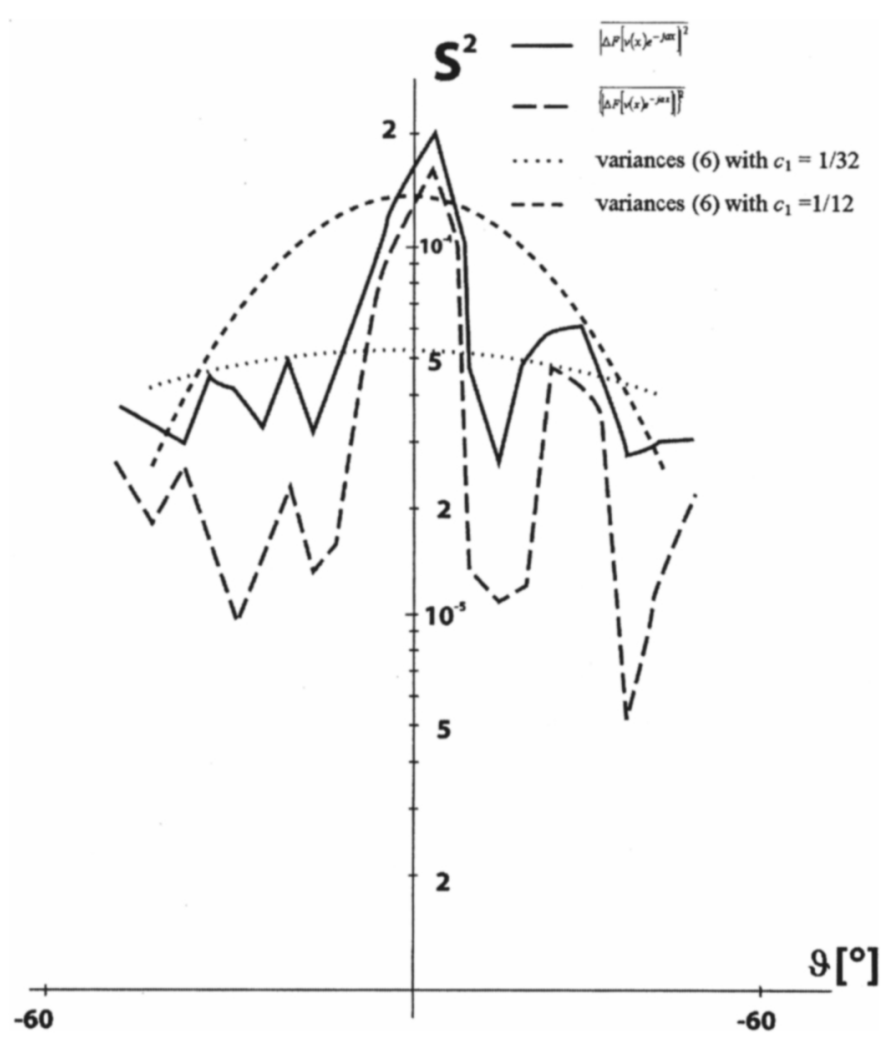

Figure 5. The variances of the measurements with a dipole, $\Delta x=7.5 \mathrm{~mm}$. 
angular spectra, $\overline{\left.\Delta F\left[v(x) e^{-j a x}\right]\right|^{2}}$, of the measurements performed with the dipole probe and with step sizes of $5 \mathrm{~mm}$ and $7.5 \mathrm{~mm}$ are shown in Figures 4 and 5 as solid lines. For comparison, the sample variances of the angular-spectrum amplitudes, $\overline{\left\{\Delta F\left[v(x) e^{-j a x}\right]\right\}^{2}}$, are plotted in Figure 4 and 5 with dashed lines. The variances were normalized to the maximum mean of the angular spectrum.

For comparison with theory, it is necessary to estimate the variances of individual error sources. The measurements (see below) indicated that the longitudinal $z$-position errors with $\sigma_{q}^{2} \approx 10^{-3}$ were the most important. The variances can be calculated using Equation (6). Assuming (see [8]) that $c_{1}=2 / N=1 / 32$, the values shown in Figures 4 and 5 by dots were obtained. Obviously, the assumption $c_{1}=2 / N$ is not correct (in fact, the correlation interval is larger).

If we assume that the correlation interval is determined by the properties of the scanner (scanning equipment), we arrive at more realistic values. If the correlation interval was $20 \mathrm{~mm}$ for non-normalized coordinates, i.e., $c_{1}=1 / 8$ for $\Delta x=5 \mathrm{~mm}$ spacing and $c_{1}=1 / 12$ for a step size of $7.5 \mathrm{~mm}$, the values obtained are as shown in Figures 4 and 5 by the shorter dashes. The values $c_{1}=1 / 8$ and $c_{1}=1 / 12$ are also not quite satisfactory. However, the agreement between the measured and calculated (Equation (6)) results using considerable simplification became worse with increasing $c_{1}$ : see $[9,14]$. Therefore, the values of Equation (6) do not agree well with the variances calculated for an increasing $c_{1}$ using Equation (11). For the limiting case $c_{1} \rightarrow \infty$, the variances are given by

$$
\overline{\left|\Delta \mathbf{F}\left[v_{q}\left(x_{1}\right) e^{-j a x_{1}}\right]\right|^{2}} \doteq \sigma_{q}^{2} \mathbf{F}\left|\left[C^{*} E\left(x_{1}\right)\right]\right|^{2} .
$$

However, this estimate is too low in the main-lobe region and too high in the sidelobe region. If the properties of the scanning equipment are not known, we can use a combination of Equation (6) for $c_{1} \geq 2 / N$ (in the sidelobe region) with Equation (12) having effect particularly in the main-lobe region. We can thus conclude that the use of Equation (6), derived with considerable simplifications, results in an approximate estimate for the variance, provided that the correlation interval, $c_{1}$, is determined by the scanning-equipment characteristics.

As mentioned above, only approximately one-third of the angular-spectrum values can be used (more accurately, those for $n=1,2, \ldots, 11$ and $n=55,56, \ldots, 64)$. When replacing the remaining values by zeros (for $n=12,13, \ldots, 54$ ) and performing the inverse Fourier transform, the approximate field amplitude and phase distributions along a line can be obtained; detailed analyses can be found in [17].

The sample mean values of the field distribution, calculated with Equation (10) using 10 values each time, are shown in Figure 6. The solid line (or the circles) represent the values determined by measurements with a dipole and $\Delta x=5 \mathrm{~mm}$ (or $\Delta x=7.5 \mathrm{~mm}$ ), and the crosses show the results of measurements with an openended waveguide. Individual cases differed, due to an improper choice of the sample interval, $\Delta x$ (measurement with the dipole

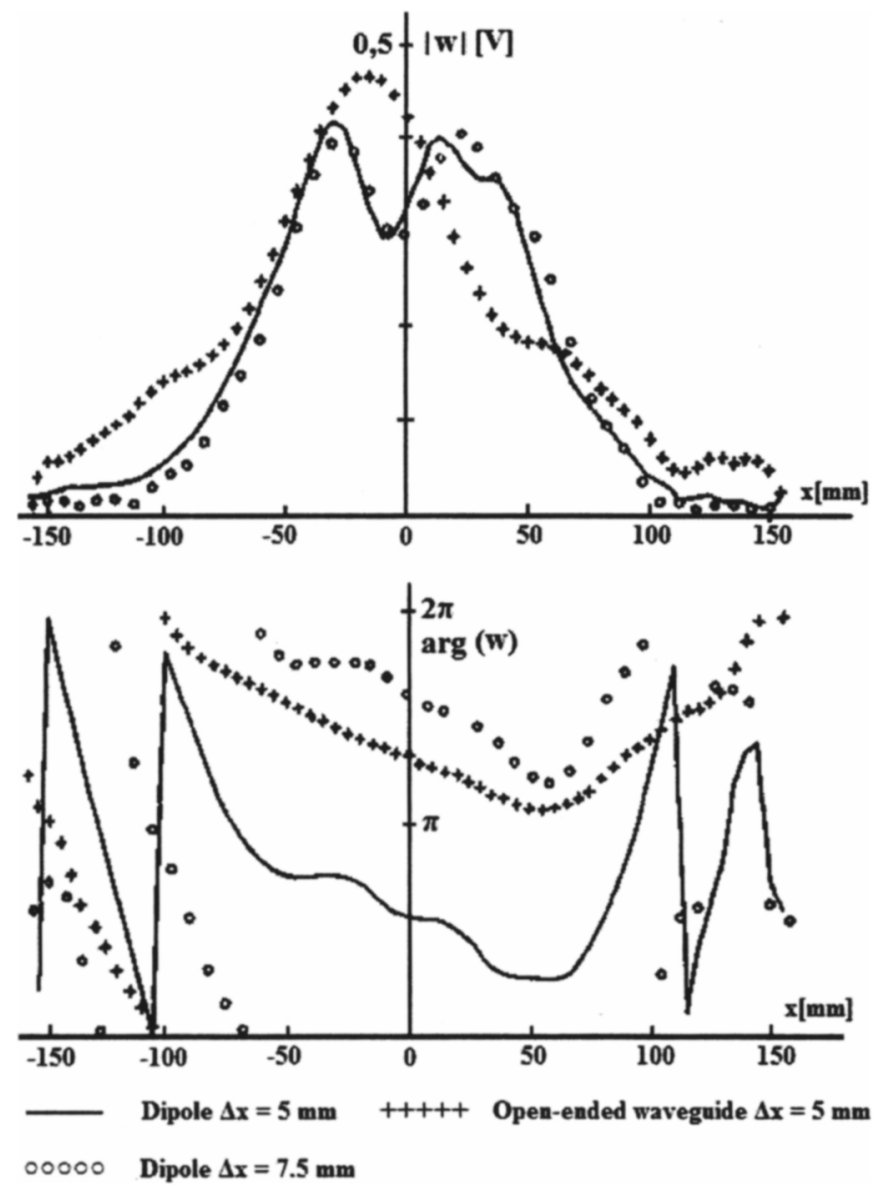

Figure 6. The sample means of the measured fields (calculated using angular spectra).

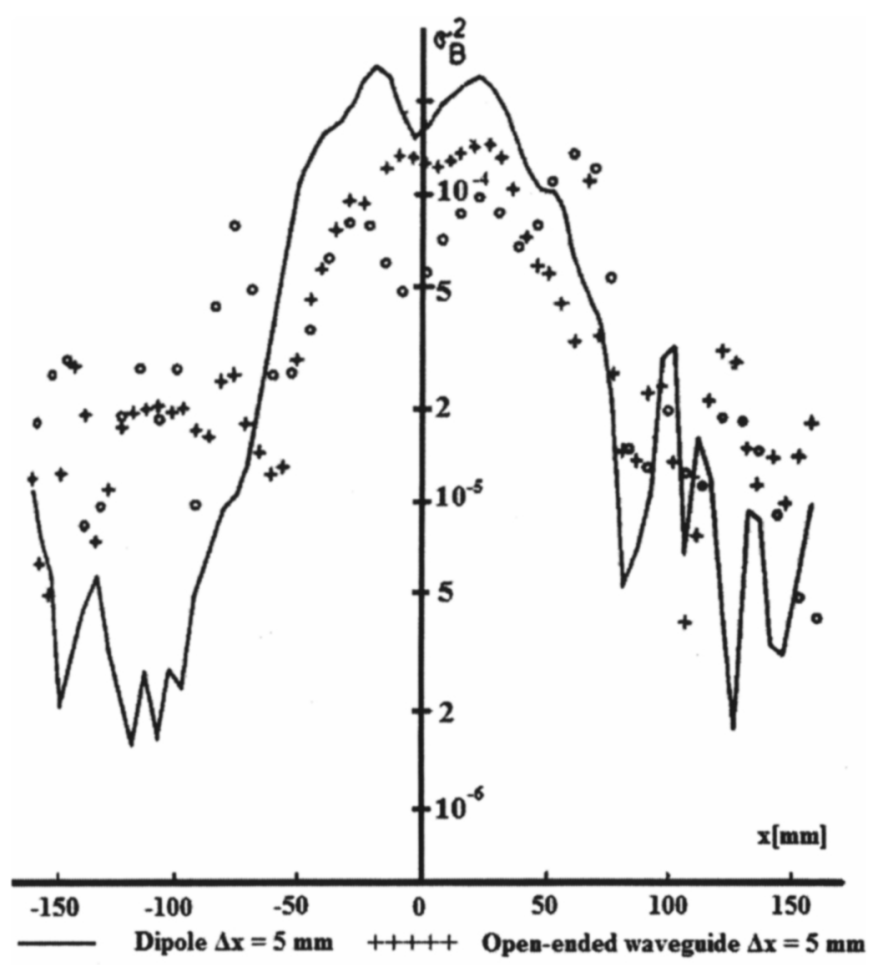

00000 Dipole $\Delta \mathbf{x}=7.5 \mathrm{~mm}$

Figure 7. The sample variances of the field-amplitude errors, $\sigma_{B}^{2}$, normalized to the maximum mean of the field amplitude. 


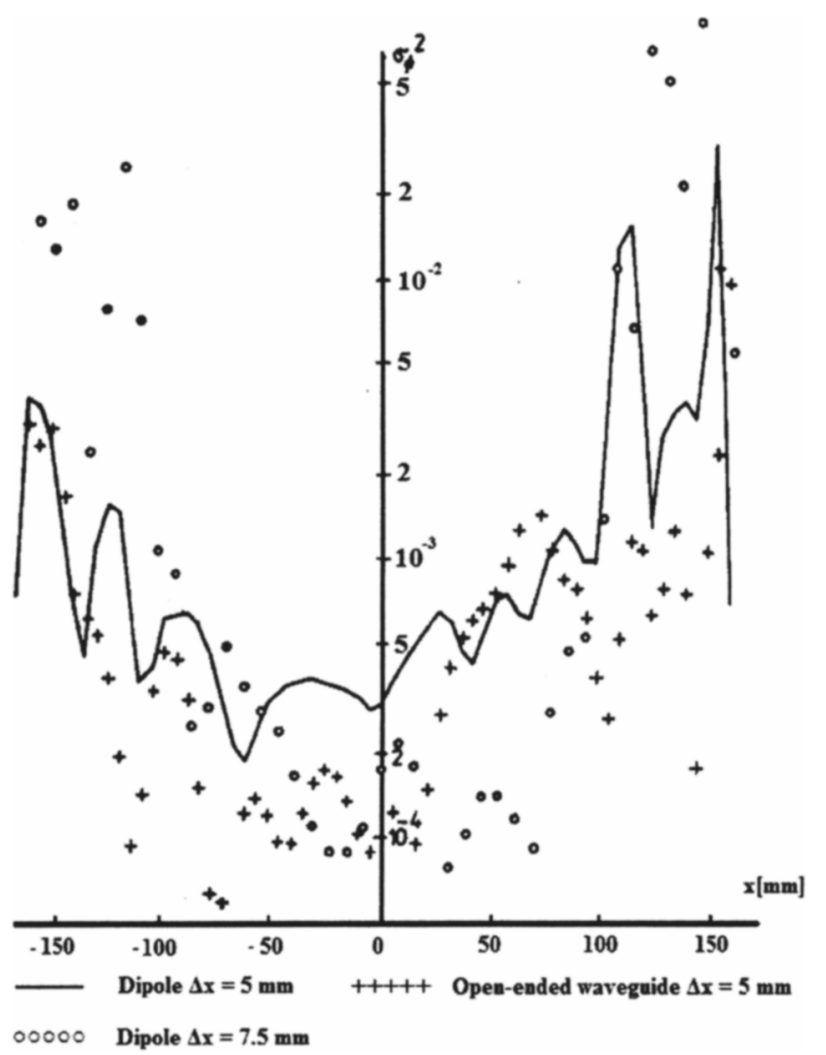

Figure 8. The sample variances of the field phase errors, $\sigma_{q}^{2}$.

\section{Numerical Simulations}

We performed numerical simulations, considering random errors as well as deterministic errors of typical problems occurring in near-field measurements $[9,12,14,16]$. Computer-generated random sequences are needed for numerical simulations. Being interested in Gaussian random sequences, the correlation sequence of Equation (2) was used. The theoretical analysis derived above used considerable simplifications. Antennas with constant amplitude and phase distributions are usually assumed, but this type of distribution does not come in question in near-field measurement. A typical field distribution is one where the phase varies quickly at the edge and the amplitude is small. Therefore, the input sequence, $E(i)$ (the near-field distribution) was considered to be

$$
E(i)=\sin ^{\alpha}(\pi i / 64) \exp \left\{j[(i-32) / 32]^{2} \beta+j \pi m / 3+j q(i)\right\},
$$

where $i=1,2, \ldots, 64 ; \quad m=i \quad$ for $i=1,2, \ldots, 11 ; \quad m=0 \quad$ for $i=12,13, \ldots, 53$ and $m=-i$ for $i=54,55, \ldots, 64 ; \alpha$ and $\beta$ are constants; and $q(i)$ are Gaussian random sequences with the correlation sequence given by Equation (2).

The measurements on a line (one-dimensional case) in the near field for both the amplitude and phase measuring method when the Fourier transform, $\mathbf{F}[E(i)]$, was determined by the FFT, and by the holographic method using Equation (4) were simulated for $\alpha=3$ and for values of $\beta$ of 0 or $2 \pi$. The near-field data were filtered (a quarter of the transformed values was only inverse transformed). Every problem was simulated with 30 realizations of $E(i)$

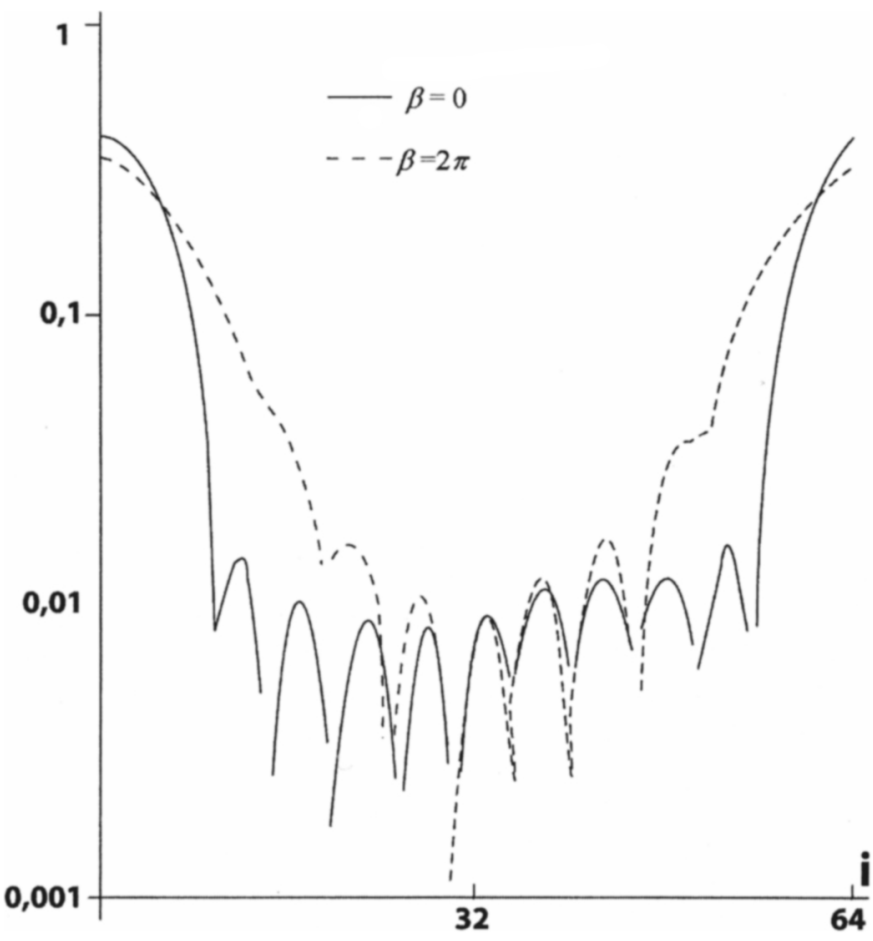

Figure 9. The mean values, $\mathbf{F}[E(i)]$, for $c_{1}=1 / 32$ and $\sigma=0.01$. and $\Delta x=7.5 \mathrm{~mm}$ ), or the influence of the probe (measurement with an open-ended waveguide).

The sample variances of the field-amplitude errors, $\sigma_{B}^{2}$, were normalized to the maximum mean of the field amplitude. The measurement results are shown in Figure 6 using a solid line (or circles) for a dipole probe and $\Delta x=5 \mathrm{~mm}$ (or $7.5 \mathrm{~mm}$ ), and using crosses for an open-ended waveguide.

The sample variances of field phase errors are plotted in Figure 7. The solid line, circles, and crosses represent the measurements with a dipole using $\Delta x=5 \mathrm{~mm}, \Delta x=7.5 \mathrm{~mm}$, and with an open-ended waveguide, respectively.

Variances determined by filtering and inverse Fourier transform present only a rough idea about the distributions and magnitudes of the measured field variances (see [9, 14]). On the other hand, these variances cannot be immediately found by holographic measurement, although this is possible in the case of amplitude and phase measurements.

As follows from Figures 7 and 8, the amplitude-error variances were of the order of $10^{-4}$, and the phase-error (positionerror) variances were of the order of $10^{-3}$. Transverse $x$-position errors caused an increase in phase errors for $|x|>100$, because the phase was not constant for $|x|>100$ but varied very quickly: see Figure 6. Because the field amplitudes were too low in this region, only a negligible influence can be assigned to the phase errors. 


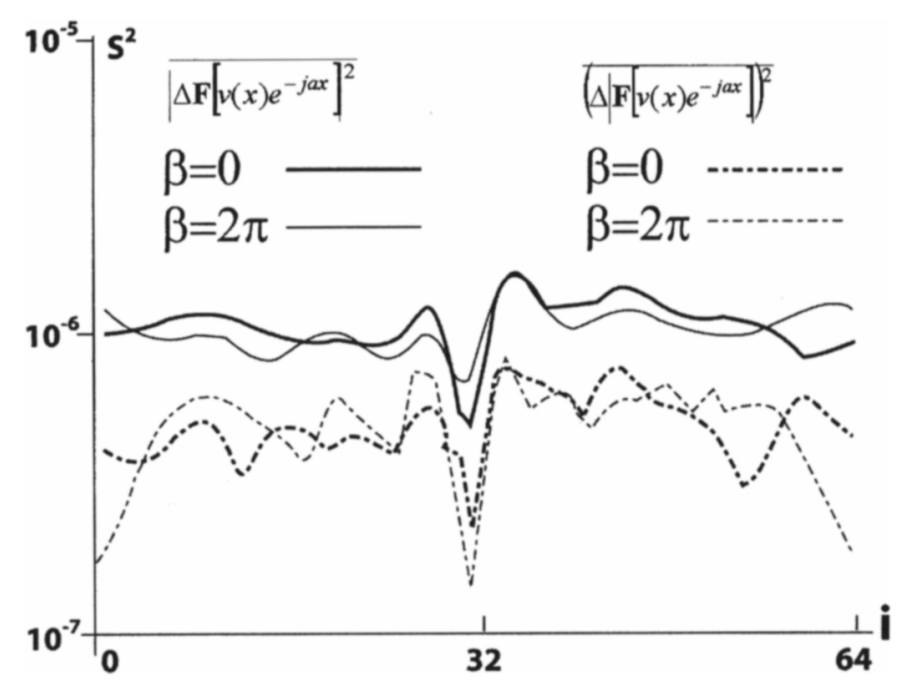

Figure 10. The sample variances of the angular spectra, $\overline{\left|\Delta \mathbf{F}\left[v(x) e^{-j a x}\right]\right|^{2}}$ and $\overline{\left(\Delta\left|F\left[v(x) e^{-j a x}\right]\right|\right)^{2}}$, for $c_{1}=1 / 32$ and $\sigma=0.01$.

Various problems for several values of $c_{1}, \beta$, and standard deviation $\sigma$ were simulated. See Figure 9 for the mean values, $\overline{\mathbf{F}[E(i)]}$. The other cases were nearly the same and they are therefore not shown. The sample variances of the angular spectrum, $\overline{\left.\Delta \mathbf{F}\left[v(x) e^{-j a x}\right]\right|^{2}}$, and those of the angular-spectrum amplitudes, $\overline{\left(\Delta\left|F\left[v(x) e^{-j a x}\right]\right|\right)^{2}}$, are plotted in Figure 10. For the sake of brevity, only some of the calculated simulations are discussed here.

Numerical simulation can be used to solve problems of measuring microwave antennas in the near field without the influence of the considerable limitations needed for an analytical solution. It was shown $[9,11]$ that theoretical estimates can be used for a good description of the variances for small intervals of $c_{1}$. With increasing $c_{1}$, the differences between the theory and numerical simulations become more significant (particularly for $c_{1}>1 / 8$ ).

\section{Conclusions}

Synthesized-reference-wave holographic techniques offer relatively simple and cost-effective measurements of antenna radiation characteristics, and reconstruction of complex aperture fields using near-field intensity-pattern measurement. We have proposed synthesized-reference-wave holography. These methods allow employing the advantages of methods for probe compensation for near-field amplitude and phase measurements for planar and cylindrical scanning, including accuracy analyses. We have published most of the results, such as a description of holographic near-field measurements using probe compensation for planar and cylindrical scanning, a comparison of holographic near-field and far-field measurements with and without probe compensation, and accuracy analyses using mathematical models considering random processes with correlation intervals, numerical simulations consid- ering random errors as well as deterministic errors, and the processing of measurement statistics.

The importance of the various near-field measurement errors depends on the antenna under test, the frequency of operation, the measurement facility, and the probe. However, the results show that the finite scan area, the $z$ position of the probe for planar scanning, receiver nonlinearities in measuring the near-field amplitude, and, sometimes, multiple reflections, are usually the most important. It is necessary to estimate the variances of individual error sources for comparison with theory. Our measurements (shown in Figures 1 to 8 ) indicated that the longitudinal $z$-position errors with $\sigma_{q}^{2} \approx 10^{-3}$ were the most important for our measurement facility. The variances can be calculated using Equation (6). We have demonstrated that the assumption $c_{1}=2 / N$ (given in the references) is not correct. The approximate estimates of the variance present reasonable results (as shown by analyses of the measurements performed by line scanning) if the correlation interval, $c_{1}$, is determined by the properties of the scanning equipment.

This paper has summarized the analyses of accuracy using mathematical models considering random processes with correlation intervals, processing of measurement statistics, and numerical simulations. It was demonstrated that the given analyses corresponded to our measurements and/or numerical simulations.

It was shown by filtering and inverse Fourier transforming that the amplitude and phase error estimates corresponded to our measured values. We can conclude that the described method is applicable both to the field measurement, performed by line scanning, and to the error analyses of this method.

\section{Acknowledgements}

The paper was supported by the Czech National Institutional Research “Theory of Transport System" No. MSM 0021627505.

\section{References}

1. A. D. Yaghjian, "An Overview of Near-Field Antenna Measurements," IEEE Transactions on Antennas and Propagation, AP34, 1, January 1986 , pp. 30-45.

2. D. Slater, "Near-Field Test Facility Design," Antenna Measurement Techniques Association Conference, 1985.

3. O. M. Bucci and M. D. Migliore, "A New Method for Avoiding the Truncation Error in Near-Field Antenna Measurements," IEEE Transactions on Antennas and Propagation, AP-54, 10, October 2006, pp. 2940-2952.

4. A. C. Newell, "Error Analysis Techniques for Planar Near-Field Measurements," IEEE Transactions on Antennas and Propagation, AP-36, 6, June 1988, pp. 754-768.

5. A. D. Yaghjian, "Upper-Bound Errors in Far-Field Antenna Parameters Determined From Planar Near-Field Measurements. Part 1: Analysis," National Bureau of Standards Technical Note 667, Boulder, October 1975. 
6. A. C. Newell and C. F. Stubenrauch, "Effect of Random Errors in Planar Near-Field Measurement," IEEE Transactions on Antennas and Propagation, AP-36, 6, June 1988, pp. 769-773.

7. A. C. Newell and D. Lee, "Application of the NIST 18 Term Error Model to Cylindrical Near-Field Antenna Measurements," Antenna Measurement Techniques Association Conference, Philadelphia, October 2000.

8. I. V. Kaplun and A. P. Kurochkin, "Analysis of Random Errors in Holographic Technique Determination of Directional Antenna Characteristics," Radiotekhnika i Elektronika, XX, 10, October 1975, pp. 2038-2045.

9. V. Schejbal, Microwave Antenna Near-Field Measurements, $\mathrm{PhD}$ Thesis, SAV Bratislava (Slovakia), 1979 (in Czech).

10. V. Schejbal, "Near-Field Antenna Measurement Using Holographic Method," Slaboproudý obzor, 39, 9, September 1978, pp. 403-407 (in Czech).

11. V. Schejbal, "Accuracy of Near-Field Antenna Measurement Using Holographic Method," Slaboproudý obzor, 41, 4, April 1980, pp. 182-186 (in Czech).

12. V. Schejbal and J. Honig, "Holographic Method of Near-Field Antenna Measurements," 10th European Microwave Conference, Warszawa, Poland,, 1980, pp. 167-171.

13. V. Schejbal and V. Kovarik, "Holography Methods for Antenna Near-Field Measurement," Tesla Electronics, 13, 2, 1980, pp. 35-39.

14. V. Schejbal, "Numerical Simulation of Near-Field Antenna Measurement," Tesla Electronics, 13, 3, 1980, pp. 67-72.

15. V. Schejbal and V. Kovarik, "Accuracy of Near-Field Antenna Measurement Using Holography," Tesla Electronics, 14, 2, 1981, pp. $48-52$.

16. V. Schejbal, "Numerical Simulations of Problems Occurring in Near-Field Antenna Measurements," 7th Colloquium on Microwave Communication, Budapest, Hungary, 1982, pp. 507-510.

17. V. Schejbal, V. Kovarik, and D. Cermak, "Synthesized-Reference-Wave Holography for Determining Antenna Radiation Characteristics," IEEE Antennas and Propagation Magazine, 50, 5, October 2008, pp. 71-83.

18. D. Smith, M. Leach, M. Elsdon, and S. J. Foti, "Indirect Holographic Techniques for Determining Antenna Radiation Characteristics and Imaging Aperture Fields," IEEE Antennas and Propagation Magazine, 49, 1, February 2007, pp. 54-67.

19. J. S. Shifrin, Problems of Statistical Antenna Theory, Part I, Moscow, S. Radio, 1970 (in Russian).

20. K. Bahadori and Y. Rahmat-Samii, "Characterization of Effects of Periodic and Aperiodic Surface Distortions on Membrane Reflector Antennas," IEEE Transactions on Antennas and Propagation, AP-53, 9, September 2005, pp. 2782-2791.

\section{Introducing the Feature Article Authors}

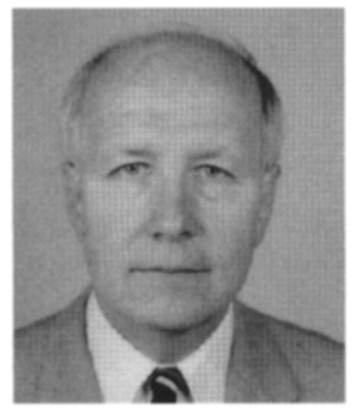

Vladimir Schejbal graduated from the Czech Technical University, Prague, in 1970. He received the $\mathrm{PhD}$ degree from the Slovak Academy of Science, Bratislava, in 1980. He was with the Radio Research Institute Opocinek, the Czech Republic (Antenna Department) from 1969 to 1993 . From 1983 to 1986 , he was on leave with the Higher Institute of Electronics (Microwave Department) Beni Walid, Libya, as a lecturer. He has been with the University of Pardubice, the Czech Republic, since 1994, now as a full professor and head of the department. He is interested in microwave antennas and propagation. He has published over 100 papers. $\mathrm{He}$ is a Senior Member of the IEEE.

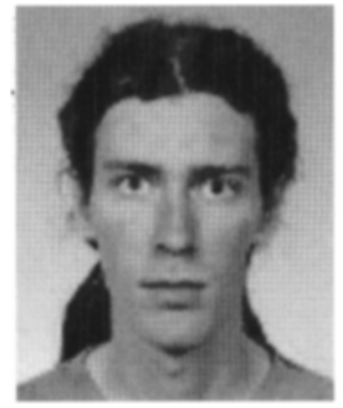

Jan Pidanic was born in 1979. He received the MS degree from the Jan Perner Transport Faculty, University of Pardubice, the Czech Republic, in 2005. He has been with the same university since 2007 , where he is now working towards his $\mathrm{PhD}$. He is interested in the propagation of electromagnetic waves, and in signal and data processing.

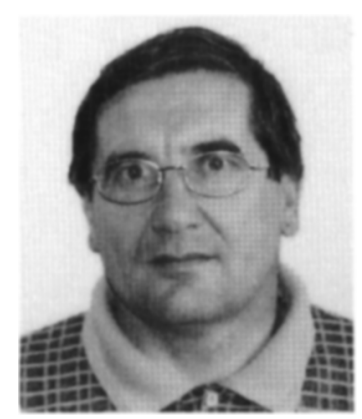

Vlastimil Kovarik completed his studies at the Pardubice High School of Electrical Engineering, the Czech Republic, in 1970. He was with the Radio Research Institute Opocinek, the Czech Republic (Antenna Department) from 1970 to 1998. He has been with Retia a.s., Pardubice, since 1998. He is interested in radar antennas and propagation. He has published over 10 papers. 


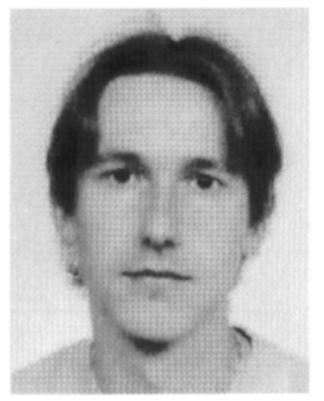

Dusan Cermak was born in 1974. He received his MS degree from the Czech Technical University in Prague in 2003, and his $\mathrm{PhD}$ from the University of Pardubice, the Czech Republic, in 2007. He has been with the University of Pardubice since 2003 as an Assistant Professor. He is interested in simulations, measurements, radar antennas, and propagation of electromagnetic waves. He has published over 20 papers. (Ais) 